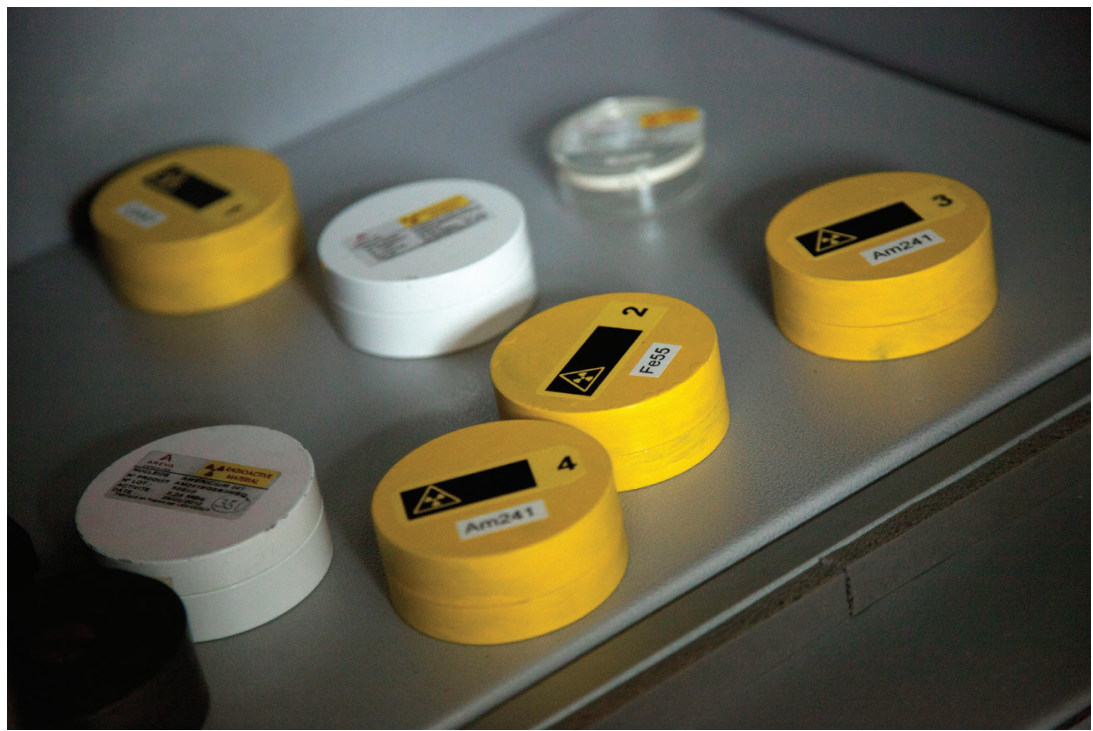

Figure 24. Sources radioactives scellées dans leur boîtier en plomb, université Toulouse III Paul-Sabatier. (c) Albin Millot/IRSN.

\title{
2.2. Cadre international
}

\subsubsection{Catégorisation des sources}

Afin de guider les États membres dans leur démarche de protection à l'égard d'un usage malveillant de sources radioactives, l'AIEA a développé une documentation spécifique dans le cadre de la « série sécurité » précédemment mentionnée (§ 1.1.5). On peut notamment citer un document de recommandations ainsi qu'un certain nombre de guides d'application des recommandations et de guides techniques.

Dans l'un de ces guides, l'AIEA propose une répartition des sources en cinq catégories numérotées de 1 (la plus dangereuse) à 5 (la moins dangereuse) fondée sur la notion de « $D$ value » ( $D$ pour danger), à savoir la valeur d'activité entraînant des effets déterministes graves. Un effet déterministe grave est défini comme un effet sanitaire dû aux rayonnements qui induit inévitablement la mort, attente à la vie ou peut conduire à une incapacité permanente diminuant la qualité de la vie. Il en a résulté le concept de source dangereuse ; ce concept a été traduit en paramètres opérationnels en calculant la quantité de matière radioactive qui conduit, pour un organisme humain, à des effets déterministes graves pour des scénarios d'exposition de personnes et pour des critères de doses donnés. 


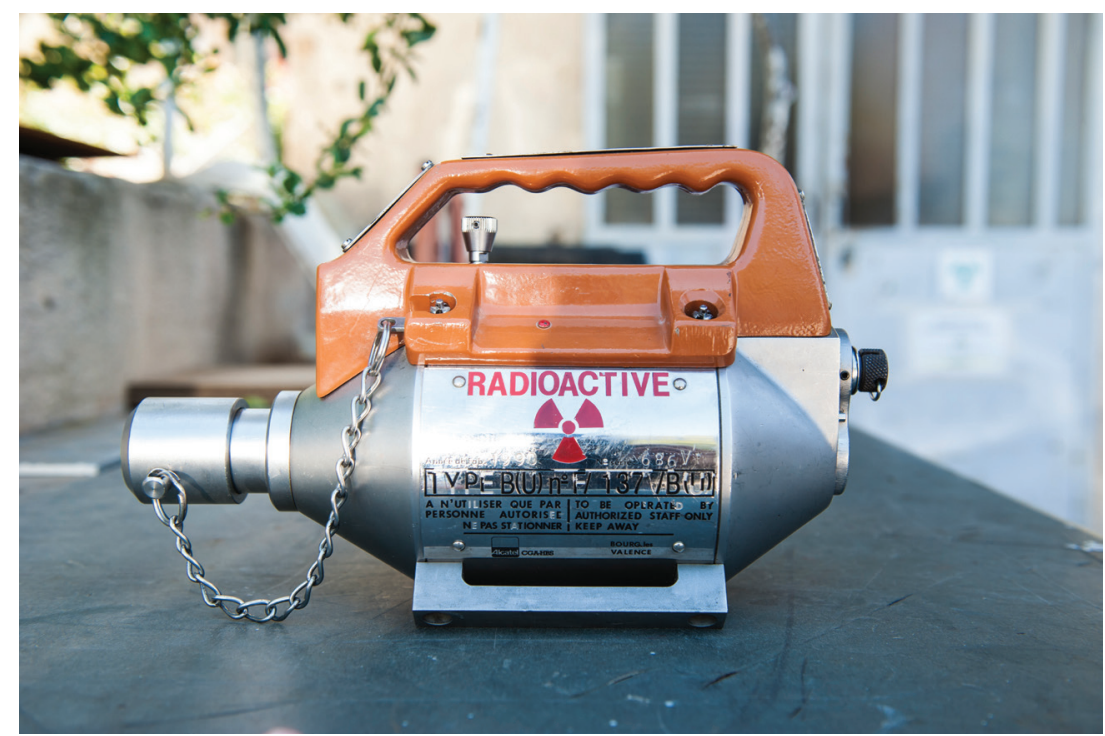

Figure 25. Projecteur de gammagraphie contenant une source radioactive, France. @ Nedim Imré/IRSN.

\subsection{2. " Code de conduite "}

Le « code de conduite » de l'AIEA sur la sûreté et la sécurité des sources radioactives vise à obtenir un niveau élevé de sûreté et de sécurité pour les sources scellées les plus dangereuses. Il présente dans ce but des orientations sur les principes de protection à mettre en œuvre et sur la réglementation en termes de sûreté, de radioprotection et de sécurité des sources (protection physique). Il a pour but de guider les États, notamment pour l'élaboration et l'harmonisation de leurs politiques et de leurs règlements sur la sûreté et la sécurité des sources radioactives. Dans ce code, seules les trois premières catégories parmi les cinq précitées font l'objet de préconisations de mesures de sécurité, en excluant les sources non scellées, les matières nucléaires et les sources intéressant la défense qui font l'objet de réglementations spécifiques.

En complément du « code de conduite », le guide de l'AIEA sur les orientations pour l'importation et l'exportation de sources radioactives préconise des notifications entre États afin de renforcer le suivi des sources des catégories 1 et 2 .

\subsubsection{Directive Euratom}

La directive européenne dite " directive Euratom » relative au contrôle des sources radioactives scellées de haute activité (SSHA) et des sources orphelines soumet certaines sources à des exigences spécifiques. Cette directive, dont la motivation principale n'est pas la sécurité des sources radioactives à l'égard d'actes de malveillance mais la radioprotection, comporte cependant des dispositions générales qui vont dans le sens de la sécurité, telles que des obligations de marquage des sources, la nécessité d'une autorisation préalable pour les détenteurs de sources radioactives, la nécessité d'une autorité compétente ayant la charge de la tenue d'un registre des détenteurs et des sources détenues... 